\title{
A UNIQUENESS THEOREM FOR RIESZ POTENTIALS
}

\author{
K. A. IZYUROV
}

\begin{abstract}
The existence is proved of a nonzero Hölder function $f: \mathbb{R} \rightarrow \mathbb{R}$ that vanishes together with its $M$. Riesz potential $f * \frac{1}{|x|^{1-\alpha}}$ at all points of some set of positive length. This result improves that of D. Beliaev and V. Havin.
\end{abstract}

\section{INTRODUCTION}

Let $\alpha$ be a real number, $0<\alpha<1$. Let $f: \mathbb{R} \rightarrow \mathbb{C}$ be a locally integrable function satisfying

$$
\int_{\mathbb{R}} \frac{|f(x)| d x}{1+|x|^{1-\alpha}}<+\infty
$$

Put

$$
\left(U_{\alpha} f\right)(t):=\int_{\mathbb{R}} \frac{f(x) d x}{|t-x|^{1-\alpha}}, \quad t \in \mathbb{R} .
$$

Under condition (1), the function $U_{\alpha} f$ is defined a.e. on $\mathbb{R}$. We call it the Riesz potential (after Marcel Riesz), and $f$ is called the density of that potential. We write $\operatorname{dom} U_{\alpha}$ (the domain of $U_{\alpha}$ ) for the set of all locally integrable functions satisying (11).

Let $V \in \mathbb{R}$ be a measurable set; we denote its length by $|V|$. The uniqueness theorem mentioned in the title states that if $f$ satisfies condition (11) and the Hölder condition with an exponent exceeding $1-\alpha$ in some neighborhood of a set $V$, and if $|V|>0$ and

$$
\left.f\right|_{V}=\left.U_{\alpha}\right|_{V}=0
$$

then $f=0$ a.e. on $\mathbb{R}$.

This theorem follows from the slightly more general "uncertainty principle" proved in [5]. The latter concerns Riesz's potentials of charges (not necessarily absolutely continuous with respect to Lebesgue measure), and the $\alpha$ 's may fail to belong to $(0,1)$; for the history of the problem and its relationship with the uniqueness problem for the Laplace equation, see also [6] and [2]. Havin [5] posed the following question: is it possible to lift the Hölder condition imposed on $f$ near $V$ in Theorem 1? Moreover, it was still unclear if there exists a nonzero continuous function $f \in \operatorname{dom} U_{\alpha}$ and a set $V$ of positive length satisfying (2).

In 2], it was shown that the answer to the latter question is in the positive. However, the continuous function $f$ constructed in 2 does not satisfy any Hölder condition.

In this paper, we build a nonzero Hölder function $f \in \operatorname{dom} U_{\alpha}$ that vanishes together with its Riesz potential $U_{\alpha} f$ on some set of positive length.

As in 2, the required function will be constructed by using the techniques of "correction", proposed by Menshov and applied to problems of potential theory in 1, 3, 4, 7,. Our progress (as compared to [2]) is based on improving the correction process with the

2000 Mathematics Subject Classification. Primary 31A15, 31A20.

Key words and phrases. Riesz potential, uncertainty principle, Hölder condition.

Partially supported by RFBR (grant no. 06-01-00313). 
help of elementary probabilistic considerations (see Lemma 5)1 It should be mentioned that we deal with complex-valued (not only real-valued!) densities $f$. This detail seems unessential, but it surprisingly simplifies the construction of the desired (real-valued) density $f$, even in the case treated in [2, where the goal was to find a continuous (not necessarily Hölder) $f$.

Our main result looks like this.

Theorem 1. There exists a nonzero function $f \in \operatorname{dom} U_{\alpha}$, a set $V \subset \mathbb{R}$ of positive length, and a positive number $r$ such that $f$ and $U_{\alpha} f$ vanish on $V$ and $f$ satisfies Hölder's condition with exponent $r$.

Remark. The function $f$ we construct is complex-valued. In order to get a real-valued density, it suffices to take the real or the imaginary part of $f$; at least one of them will not be identically zero.

I am grateful to V. Havin for introducing me to the problem and for useful discussions.

$$
\S 1 \text {. The operator } W_{\alpha}
$$

We shall need an operator inverse (in a sense) to $U_{\alpha}$. For $g \in C_{0}^{\infty}(\mathbb{R})$, we put

$$
\begin{gathered}
\left(V_{\alpha} g\right)(t):=\frac{1}{\alpha} \int_{\mathbb{R}} g(x) \frac{\operatorname{sgn}(t-x)}{|t-x|^{\alpha}} d x, \\
\left(W_{\alpha} g\right)(t)=\left(\left(V_{\alpha} g\right)(t)\right)^{\prime}, \quad t \in \mathbb{R} .
\end{gathered}
$$

Let $g$ be a function defined on $\mathbb{R}$, and let $\lambda>0, \varepsilon>0$. We denote $\left(C_{\lambda} g\right)(x)=g(\lambda x)$, $g_{\varepsilon}=\frac{1}{\varepsilon} C_{1 / \varepsilon} g$. In the next lemma we list some properties of $W$.

Lemma 1. 1) $W_{\alpha}\left(C_{0}^{\infty}(\mathbb{R})\right) \subset \operatorname{dom} U_{\alpha}$;

2) $U_{\alpha} W_{\alpha} g=c g, g \in C_{0}^{\infty}(\mathbb{R})$;

3) $W_{\alpha} C_{\lambda}=\lambda^{\alpha} C_{\lambda} W_{\alpha}$;

4) $\alpha W_{\alpha} g=-g^{\prime} * \frac{\operatorname{sgn} x}{|x|^{\alpha}}$ (we use $*$ for the convolution on $\left.\mathbb{R}\right)$;

5) $\left(W_{\alpha} g\right)(t)=\left(g *|x|^{-\beta)}(t), g \in \operatorname{dom} W_{\alpha}, t \notin \operatorname{supp} g\right.$.

Here and in what follows, $\beta=\alpha+1$. The statements of this lemma are either wellknown (statement 2)), or obvious (statements 1), 3)-5)). See, e.g., [2, p. 226].

We use the following notation: $I=\left(-\frac{1}{2}, \frac{1}{2}\right)$; if $Q$ is a bounded interval, then $c_{Q}$ is its center.

We write $\phi(t)$ for a "smooth cap": $\phi \in C^{\infty}, \operatorname{supp} \phi \subset I, \int_{I} \phi=1, \phi \geq 0$.

In the proof we shall fix positive numbers $p$ and $\lambda$. With a function $h: \mathbb{R} \rightarrow \mathbb{C}$, we associate its "reducing to the interval $Q$ ", putting $h_{Q}(t):=h\left(\frac{t-c_{Q}}{|Q| \lambda}\right), t \in \mathbb{R}$. Finally, let $M_{Q}(h):=\left(\frac{1}{|Q|} \int_{Q}|h|^{p}\right)^{1 / p}$.

\section{$\S 2$. MAin Lemma}

The mail tool in the proof of Theorem 1 will be Lemma 4. First, we prove the auxiliary Lemmas 2 and 3. We establish the existence of functions with specific quantitative properties. These functions will serve as "building blocks" for our construction. The following assertion is crucial for us: for some constant $B<0$ and some $p>0$, there exists a function $h \in C_{0}^{\infty}$ with arbitrarily short support satisfying $\int_{\mathbb{R}}\left(\left|1-W_{\alpha} h\right|^{p}-1\right)<B$. The meaning of this fact is that it allows us to control both the length of the support of $h$ and the "size" of the potential $W_{\alpha}$ of $h$. We shall reshape this assertion to a form convenient for our purposes.

The function $h$ will be made from the "smooth cap" $\phi$ via an appropriate scaling.

\footnotetext{
${ }^{1}$ I am grateful to S. Smirnov for useful discussions concerning this lemma.
} 
For $\varepsilon>0$ and $t \in \mathbb{R}$, let

$$
F^{[\varepsilon]}(t):=\left(W_{\alpha} \phi_{\varepsilon}\right)(t), \quad F^{[0]}(t):=|t|^{-\beta} .
$$

Lemma 2. If a positive number $p$ is sufficiently small, then

$$
J(p):=\int_{\mathbb{R}}\left(\left|1-F^{[0]}\right|^{p}-1\right) d x<0 .
$$

Proof. Let $L:=\int_{\mathbb{R}} \log \left|1-F^{[0]}\right|$. Then, since $\left(a^{p}-1\right) / p$ is monotone in $p$ for any $a>0$ and converges to $\log a$ as $p \rightarrow 0$, we have $\lim _{p \backslash 0} \frac{J(p)}{p}=L$ (observe that || $1-\left.F^{[0]}(t)\right|^{p}-1 \mid \leq$ $c|t|^{\beta}$ if $|t|$ is large, and that if $p<1 / \beta$, then the integral $J(p)$ converges at zero). But $L$ can be computed explicitly: $L=2 \pi \cot \frac{\pi}{\beta}<0$. The computation can be found, e.g., in [2, p. 233].

From now on, the number $p$ found in the preceding lemma will be fixed.

In the next lemma we pass from $F^{[0]}$ (the potential $W_{\alpha}$ of the delta function) to the potential of some specific function $\phi_{\varepsilon}$. We also introduce a "small complex rotation": we multiply $\phi_{\varepsilon}$ by $e^{i \theta}$ with small $\theta$. This will lead to some technical simplifications in what follows.

Lemma 3. There exist numbers $B<0, \theta_{0}>0$, and $\varepsilon_{0}>0$ such that if $0 \leq \theta \leq \theta_{0}$ and $0 \leq \varepsilon<\varepsilon_{0}$, then

$$
J(\varepsilon, \theta):=\int_{\mathbb{R}}\left(\left|1-e^{i \theta} F^{[\varepsilon]}\right|^{p}-1\right)<B .
$$

Proof. Using the homogeneity property of $W_{\alpha}$ (item 3 ) of Lemma 1), we get

$$
\left|F^{[\varepsilon]}(t)\right| \leq C(\alpha) \min \left(\frac{1}{\varepsilon^{\beta}}, \frac{1}{|t|^{\beta}}\right), \quad t \in \mathbb{R} .
$$

Clearly, $F^{[\varepsilon]}$ converges to $F^{[0]}$ pointwise as $\varepsilon \rightarrow 0$. The Lebesgue dominated convergence theorem yields

$$
\lim _{\varepsilon \searrow 0} J(\varepsilon, 0)=J(p)
$$

We choose $B<0$ so that $J(\varepsilon, 0)<2 B$ for $\varepsilon \in\left(0, \varepsilon_{0}\right)$. We shall prove that $J(\varepsilon, \theta) \stackrel{\theta \rightarrow 0}{\longrightarrow}$ $J(\varepsilon, 0)$ uniformly in $\varepsilon$. Indeed,

$|J(\varepsilon, \theta)-J(\varepsilon, 0)|=\left|\int_{\mathbb{R}}\right| 1-\left.e^{i \theta} F^{[\varepsilon]}\right|^{p}-\left|1-F^{[\varepsilon]}\right|^{p}|\leq| \int_{|x|>\theta^{-p / 2}}|+| \int_{|x|<\theta^{-p / 2}} \mid=: J_{1}+J_{2}$.

We have

$$
J_{1}=\left|\int_{|x|>\theta^{-p / 2}}\right| 1-\left.e^{i \theta} F^{[\varepsilon]}\right|^{p}-\left|1-F^{[\varepsilon]}\right|^{p}|\leq 2 C| \int_{|x|>\theta^{-p / 2}}|t|^{-\beta} \mid \stackrel{\theta \rightarrow 0}{\longrightarrow} 0,
$$

because the integral converges at infinity (and does not depend on $\varepsilon$ ). Next, using the inequality $\left|a^{p}-b^{p}\right| \leq|a-b|^{p}$ for $a>0, b>0$ and $p \in(0,1)$, we get

$$
\begin{aligned}
J_{2} & =\left|\int_{|x|<\theta^{-p / 2}}\right| 1-\left.e^{i \theta} F^{[\varepsilon]}\right|^{p}-\left|1-F^{[\varepsilon]}\right|^{p} \mid \\
& =\left|\int_{|x|<\theta^{-p / 2}}\right| e^{-i \theta}-\left.F^{[\varepsilon]}\right|^{p}-\left|1-F^{[\varepsilon]}\right|^{p} \mid \\
& \leq \int_{|x|<\theta^{-p / 2}}\left|e^{-i \theta}-1\right|^{p} \leq \int_{|x|<\theta^{-p / 2}} \theta^{p} \stackrel{\theta \rightarrow 0}{\longrightarrow} 0 .
\end{aligned}
$$

Thus, $J_{1}+J_{2} \stackrel{\theta \rightarrow 0}{\longrightarrow} 0$ uniformly in $\varepsilon \in\left(0, \varepsilon_{0}\right)$, and the lemma is proved. 
Now we are ready to formulate Lemma 4, the main ingredient of the further construction. We replace the entire line $\mathbb{R}$ (occurring in Lemma 3) by a bounded interval and the constant 1 by an arbitrary function with small oscillation. Also, we introduce a (small) positive parameter $\lambda$. This parameter will be in charge of the smallness of the potential $W_{\alpha}$ of the correcting term far from the interval on which we correct. Denote $\gamma(\lambda):=(1+B \lambda / 2)^{1 / p}$, where $B$ is as in Lemma 3 . Observe that $0<\gamma(\lambda)<1$ for all sufficiently small positive $\lambda$.

If $f$ is a function defined on some interval $Q$, we define

$$
\operatorname{osc}_{Q} f:=\sup _{x, y \in Q}(|f(x)-f(y)|)
$$

(the oscillation of $f$ on $Q$ ).

Lemma 4. There exist numbers $\theta>0, \lambda_{0}>0$, and $\varepsilon_{0}>0$ such that for any positive $\lambda<\lambda_{0}$ there is a number $\kappa>0$ with the following property: if $0<\varepsilon<\varepsilon_{0}, Q$ is any bounded interval, and a continuous complex-valued function $h$ satisfies

$$
\operatorname{osc}_{Q} h \leq \kappa\left|h\left(c_{Q}\right)\right|,
$$

then

1) $M_{Q}\left(h-h\left(c_{Q}\right) e^{i \theta} F_{Q}^{[\varepsilon]}\right) \leq \gamma(\lambda)\left|h\left(c_{Q}\right)\right|$;

2) $\frac{\theta}{2}|h(t)| \leq\left|h(t)-h\left(c_{Q}\right) e^{i \theta} F_{Q}^{[\varepsilon]}(t)\right| \leq \frac{C}{\varepsilon^{\beta}}|h(t)|, t \in Q$.

Recall that $F_{Q}^{[\varepsilon]}(t)=F^{[\varepsilon]}\left(\frac{t-c_{Q}}{|Q| \lambda}\right)$.

Proof. Let $\varepsilon_{0}$ and $\theta$ be as in Lemma 3. First, we get the estimate in item 2):

$$
\begin{aligned}
\mid h(t) & -h\left(c_{Q}\right) e^{i \theta} F_{Q}^{[\varepsilon]} \mid \\
& \geq\left|h\left(c_{Q}\right)\right|\left|1-e^{i \theta} F_{Q}^{[\varepsilon]}(t)\right|-\left|h(t)-h\left(c_{Q}\right)\right| \geq\left|h\left(c_{Q}\right)\right| \frac{3 \theta}{4}-\kappa\left|h\left(c_{Q}\right)\right| \\
& =\left|h\left(c_{Q}\right)\right|\left(\frac{3 \theta}{4}-\kappa\right) \geq|h(t)| \frac{1}{1+\kappa}\left(\frac{3 \theta}{4}-\kappa\right) \geq|h(t)| \frac{\theta}{2}
\end{aligned}
$$

for any sufficiently small $\kappa$. We use the elementary inequality $\operatorname{dist}\left(1,\left\{r e^{i \theta}: r \in \mathbb{R}\right\}\right)=$ $\sin \theta \geq \frac{3 \theta}{4}$ provided $\theta>0$ is small.

Clearly, the right-hand inequality in 2) follows from (3).

Now we prove 1 ). We have

$$
\begin{aligned}
\left(M_{Q}\left(h-h\left(c_{Q}\right) e^{i \theta} F_{Q}^{[\varepsilon]}\right)\right)^{p} & \leq\left(M_{Q}\left(h-h\left(c_{Q}\right)\right)\right)^{p}+\left|h\left(c_{Q}\right)\right|^{p}\left(M_{Q}\left(1-e^{i \theta} F_{Q}^{[\varepsilon]}\right)\right)^{p} \\
& \leq\left(\operatorname{osc}_{Q} h\right)^{p}+\left|h\left(c_{Q}\right)\right|^{p}\left(M_{\lambda^{-1}}\left(1-e^{i \theta} F^{[\varepsilon]}\right)\right)^{p} .
\end{aligned}
$$

Observe that

$$
\begin{aligned}
\left(M_{\lambda^{-1} I}\left(1-e^{i \theta} F^{[\varepsilon]}\right)\right)^{p} & =1+\lambda \int_{\lambda^{-1} I}\left(\left|1-e^{i \theta} F^{[\varepsilon]}\right|^{p}-1\right) \\
& =1+\lambda \int_{\mathbb{R}}\left(\left|1-e^{i \theta} F^{[\varepsilon]}\right|^{p}-1\right)-\lambda \int_{\mathbb{R} \backslash \lambda^{-1} I}\left(\left|1-e^{i \theta} F^{[\varepsilon]}\right|^{p}-1\right) .
\end{aligned}
$$

Using estimate 3$)$, we see that

$$
\left|\lambda \int_{\mathbb{R} \backslash \lambda^{-1} I}\left(\left|1-e^{i \theta} F^{[\varepsilon]}\right|^{p}-1\right)\right| \leq C \lambda^{\alpha+1}=o(\lambda), \quad \lambda \longrightarrow 0 .
$$


Thus, if $\lambda$ is sufficiently small, we have $M_{\lambda^{-1} I}\left(1-e^{i \theta} F^{[\varepsilon]}\right) \leq 1+B \lambda / 2<1$. Therefore,

$$
\begin{aligned}
M_{Q}\left(h-h\left(c_{Q}\right) e^{i \theta} F^{[\varepsilon]}\right)^{p} & \leq\left|h\left(c_{Q}\right)\right|^{p}\left(1+\left(\frac{\operatorname{osc}_{Q}(h)}{h\left(c_{Q}\right)}\right)^{p}+2 B \lambda / 3\right) \\
& \leq\left|h\left(c_{Q}\right)\right|^{p}\left(1+\kappa^{p}+2 B \lambda / 3\right) .
\end{aligned}
$$

If $\kappa$ is sufficiently small, then $\left(1+\kappa^{p}+2 B \lambda / 3\right)<1+B \lambda / 2$. The lemma is proved.

Remark 1. Careful examination of the above proof shows that we can take $\kappa$ equal to $\min \left(\left(\frac{|B| \lambda}{2}\right)^{1 / p}, \frac{\theta}{8}\right)$ if $\theta$ is not too large.

Remark 2 . The left-hand inequality in 2 ) is precisely the reason for passing to complexvalued functions. Such an estimate cannot be obtained for real-valued functions.

Remark 3. For this moment, we have fixed the parameters $p$ and $\theta$. In what follows, the constants viewed as depending on $\alpha$ may also depend on these parameters. Later we shall fix an appropriate $\lambda$ and, with it, $\kappa$ and $\gamma$.

\section{§3. General idea of the construction}

Now we describe the plan of constructing $f$ and $V$ (see Theorem 1). We shall build a sequence of functions $g_{n}, g_{n}=g_{n-1}-r_{n-1}$, and a monotone decreasing sequence of sets $V_{n} \subset I$ with the following properties:

1) the nonzero function $g_{1}$ belongs to $C_{0}^{\infty}$, and supp $g_{1} \subset \mathbb{R} \backslash I$;

2) $r_{k} \in C_{0}^{\infty}$ and supp $r_{k} \subset I$ for all $k \in \mathbb{N}$;

3) $\sum_{k=1}^{\infty}\left|\operatorname{supp} r_{k}\right|<\frac{1}{4}$

4) $\left|\bigcap_{k=1}^{\infty} V_{n}\right|>\frac{3}{4}$;

5) $\int_{V_{n}}\left|f_{n}\right|^{p} \stackrel{n \rightarrow \infty}{\longrightarrow} 0$, where $f_{n}:=W_{\alpha} g_{n}$ and $p$ is the positive number fixed above;

6 ) the sequences $g_{n}$ and $f_{n}$ converge uniformly on $\mathbb{R}$ to some continuous functions $g$ and $f$, respectively, and $g=U_{\alpha} f$.

Put $V:=\bigcap_{k=1}^{\infty} V_{n}$ and $V^{\prime}:=\{x \in I: g(x)=0\}$. Properties 1), 3), and 4) show that $\left|V^{\prime}\right|>\frac{3}{4}$ and $|V|>\frac{3}{4}$. Therefore, $\left|V \cap V^{\prime}\right|>\frac{1}{2}$. Properties 5) and 6) imply that $\left.f\right|_{V}=0$. Finally, using property 2), we conclude that $\left.g\right|_{\mathbb{R} \backslash I}=\left.g_{1}\right|_{\mathbb{R} \backslash I}$, so that the function $g$ is not identically 0 . Hence, the set $V \cap V^{\prime}$ and the function $f$ satisfy all conditions of Theorem 1 except (possibly) the Hölder condition.

Now we describe the structure of the sets $V_{n}$ and the correcting terms $r_{k}$ in more detail. We introduce a sequence of positive numbers $\left\{\delta_{n}\right\}_{1}^{\infty}$ such that $\delta_{1}=1$ and $\frac{\delta_{n}}{\delta_{n+1}} \in \mathbb{N}$. We denote by $H_{n}$ the partition of the interval $I$ into intervals of length $\delta_{n}$. The set $V_{n}$ will be obtained as the union $\bigcup_{Q \in G_{n}} Q$, where $G_{n}$ is some subset of $H_{n}$. Roughly speaking, the set $G_{n}$ consists of all intervals on which correction has not yet been finished; in particular, for all $k>n$ we have supp $r_{k} \subset V_{n}$.

We fix a sequence $\left\{\varepsilon_{n}\right\}_{n=1}^{\infty}$ of positive numbers such that $\sum_{n=1}^{\infty} \varepsilon_{n}<\frac{1}{4}$ and, moreover, the $\varepsilon_{n}$ decay not very fast: $\varepsilon_{n}^{-1}=O\left(n^{m}\right)$ for some $m>0$. These numbers will control the lengths of the supports of $r_{n}$ : $\left|\operatorname{supp} r_{n}\right| \leq \varepsilon_{n}$ for all $n$.

We also demand that supp $g_{1} \in\left(\frac{1}{2}, \frac{3}{2}\right)$ and, moreover, $f_{1}(t) \neq 0$ for all $t \in I$. We can take, for example, $g_{1}:=\phi(x-1)$.

Next, we choose a subset $G_{n}^{g} \subset G_{n}$ (to be defined later) and put

$$
r_{n}:=\sum_{Q \in G_{n+1}^{g}}\left(\lambda \delta_{n+1}\right)^{\alpha} f_{n}\left(c_{Q}\right)\left(\phi_{\varepsilon_{n}}\right)_{Q} e^{i \theta} .
$$

Then

$$
W_{\alpha} r_{n}=\sum_{Q \in G_{n+1}^{g}} f_{n}\left(c_{Q}\right) F_{Q}^{\left[\varepsilon_{n}\right]} e^{i \theta}
$$


Note that, under such a definition, condition 3) will be ensured by the choice of the sequence $\varepsilon_{n}$ as above.

The idea is that if $\delta_{n+1}$ is sufficiently small, then on each interval $Q \in G_{n+1}^{g}$ the oscillation of $f_{n}$ is small (estimate (44) is valid), and we can apply Lemma 4 with $f_{n}$ in the role of $h$. Combined with the observation that the functions $F_{Q}^{[\varepsilon]}$ decay sufficiently fast far from $Q$, Lemma 4 allows us, putting $V_{n}^{g}:=\bigcup_{Q \in G_{n}^{g}} Q$, to prove the estimate

$$
\int_{V_{n+1}^{g}}\left|f_{n+1}\right|^{p} \leq \eta \int_{V_{n}^{g}}\left|f_{n}\right|^{p}
$$

with some $\eta \in(0,1)$. If the sets $G_{n}^{g}$ are chosen appropriately (at each step they occupy a large part of $G_{n}$ ), this leads to an estimate for the integral over the entire set $V_{n}$ :

$$
\int_{V_{n}}\left|f_{n}\right|^{p}=O\left(\eta^{\frac{n}{2}}\right)
$$

This ensures condition 5).

Remark 1. The choice of $G_{n}$ (reduction of $V_{n}$ at each step) allows us to make the functions $f_{n}$ converge not only in the sense of $L^{p}(I)$, but uniformly; in particular, we obtain the estimate $\left|f_{n}\left(c_{Q}\right)\right|=O\left(\eta^{\prime n}\right), Q \in G_{n}$, where $\eta^{\prime} \in(0,1)$.

Remark 2. If we did not worry about controlling the modulus of continuity, we could take $G_{n}^{g}:=G_{n}$. Then (7) automatically follows from (6), and the entire construction becomes simpler. Unfortunately, in order to get Hölder's condition, we are forced to pick out the set $G_{n}^{g}$ at each step (this is a set of intervals where the oscillation of $f_{n}$ is especially small) and to perform a correction only there.

\section{§4. Remarks on estimation of the modulus of CONTinuity}

In this section, we explain (not quite rigorously) our plan of obtaining estimates for the modulus of continuity of $f$.

We use the following simple fact: if a sequence of functions $h_{n}$ converges on $\mathbb{R}$ to a function $h$, and $\left|h_{n}-h\right| \leq C_{1} \eta_{1}^{n},\left|h_{n}^{\prime}\right| \leq C_{2} R^{n}$ (here $\eta_{1} \in(0,1), R>1$ ), then $h$ satisfies the Hölder condition with the exponent $\log \eta_{1} / \log \frac{\eta_{1}}{R}$.

The condition $\left|f_{n}-f\right| \leq C_{1} \eta_{1}^{n}$ will follow from Remark 1 at the end of $\S 2$ (and, in fact, from estimate (7D). In estimation of the derivative $f_{n}^{\prime}(t)$ of $f_{n}$, the main role is played by the last added term $W_{\alpha} r_{n-1}$, or more precisely, by the building block $f_{n-1}\left(c_{Q_{t}}\right) F_{Q_{t}}^{\left[\varepsilon_{n-1}\right]} e^{i \theta}$, where the interval $Q_{t} \in H_{n}$ is determined by the condition $t \in Q_{t}$. From the homogeneity properties of $W_{\alpha}$ we can deduce that, for $Q \in H_{n}$,

$$
\left|\left(F_{Q}^{\left[\varepsilon_{n-1}\right]}\right)^{\prime}(t)\right| \leq \frac{c}{\varepsilon^{-\beta-1} \lambda \delta_{n}}, \quad t \in \mathbb{R}
$$

Thus, say, on $V_{n}$ we can get the estimate

$$
\left|f_{n}^{\prime}\right| \leq \frac{C f_{n}\left(c_{Q_{t}}\right)}{\delta_{n} \varepsilon_{n-1}^{\beta+1}} .
$$

Therefore, everywhere we have

$$
\left|f_{n}^{\prime}\right| \leq \frac{C \eta^{\prime n}}{\delta_{n} \varepsilon_{n-1}^{\beta+1}} .
$$

This means that the resulting function $f$ would be Hölder should the numbers $\delta_{n}^{-1}$ grow not faster than some geometric series, in other words, should we split the interval $Q \in H_{n}$ each time into the same number of parts. On the other hand, the exponent 
$\log \eta_{1} / \log \frac{\eta_{1}}{R}$ tends to zero as $R \rightarrow \infty$, whence it is clear that if $\frac{\delta_{n}}{\delta_{n+1}} \rightarrow \infty$ as $n \rightarrow \infty$, then we are unable to prove Hölder's condition with any exponent.

It is not hard to see, however, that if we use the natural estimate (9) in order to define $\delta_{n+1}$ (recall that $\delta_{n+1}$ should be taken small because we need an estimate for the oscillation of $f_{n}$ in order to use Lemma 4, see condition (44)), then the presence of the growing factor $\varepsilon_{n-1}^{-\beta-1}$ shows that we must take $\delta_{n+1} / \delta_{n}$ tending to zero to ensure (4). Therefore, we need finer estimates of the modulus of continuity of $f_{n}$ that are valid, however, not on the entire set $V_{n+1}$, but on some "good" part $V_{n+1}^{g}$ of it.

Note that the building block $F_{Q}^{\left[\varepsilon_{n}\right]}$ and its derivative $\left(F_{Q}^{\left[\varepsilon_{n}\right]}\right)^{\prime}$ are large in modulus (of the order of $\varepsilon_{n}^{-\beta}$ and $\delta_{n+1}^{-1} \varepsilon_{n}^{-\beta-1}$, respectively) only near the center of $Q$; outside the interval of length $\tau|Q|$ and with the same center, where $0<\tau<1$, we have $\left|F_{Q}^{\left[\varepsilon_{n}\right]}\right| \leq C$ and $\left|\left(F_{Q}^{\left[\varepsilon_{n}\right]}\right)^{\prime}\right| \leq C \delta_{n+1}^{-1}$.

The idea arises to remove this "bad" central part of $Q$ and to correct on the remaining part only. Unfortunately, if we drop it forever, this will mean that at each step we remove from $V_{n}$ a subset of length $\tau\left|V_{n}\right|$, and the intersection $\bigcap_{n \in \mathbb{N}} V_{n}$ will have zero length.

Therefore, for each interval of the partition $H_{n}$ we introduce a system of its "bad" subsets, and on each of them we shall "make a pause", namely, shall not correct during the next few steps, until the partition $H_{n+k}$ becomes so fine that the estimates of $f_{n}$ and its derivative become satisfactory (the requirements on these estimates become weaker as $n$ grows). The pause duration depends on the distance between the corresponding subset and the center of the interval, i.e., on how "bad" $f_{n}$ is on that subset. Accordingly, $G_{n+1}$ splits into two parts: $G_{n+1}^{g}$, where the correction is made immediately, and $G_{n+1}^{d}$, where we refrain from doing anything for the time being 2

After that, we estimate a number of the intervals in $G_{n}$ such that more than half of their "ancestors" in $G_{k}, k=0,1, \ldots, n-1$, belong to $G_{k}^{d}$. It turns out that there are only a few of them (if $G_{k}^{d}$ is a small part of $G_{k}$ for each $k$ ), and we drop them out. For the remaining intervals, we prove an estimate like (7), using Lemma 4 and the fast decay of $F_{Q}^{\left[\varepsilon_{n}\right]}$ far from $Q$.

\section{$\S 5$. Definition of The SETS $G_{n}^{g}$}

To complete the construction, we must define the sequence $\delta_{n}$ and the sets $G_{n}$ and $G_{n}^{g}$. We shall need a number of estimates, which will depend on the choice of the "good" subsets $G_{n}^{g}$ in $G_{n}$, but not on the choice of the sets $G_{n}$ themselves. These estimates will be proved in $\S \S 6,7$, and 8 . Later, in $\S 9$, we shall describe the way to choose $G_{n}$.

Let $\delta$ be a positive parameter such that $\delta^{-1} \in \mathbb{N}$, and let $\delta_{n}:=\delta^{n}$.

Next, let $\tau \in(0,1)$. We assume that $\tau^{-1} \in \mathbb{N}$, and moreover, $\tau / \delta \in \mathbb{N}$. We use the following notation: if $a>0$ and $Q$ is a bounded interval, then $Q[a]:=Q \backslash Q^{\prime}$, where $Q^{\prime}$ is the interval of length $a|Q|$ centered at the center of $Q$.

Now we are ready to define the set $G_{n+1}^{g}$. An interval $Q \in G_{n+1}$ belongs to $G_{n+1}^{g}$ if and only if for any $k=0,1, \ldots, n-1$ the condition $Q \subset Q^{\prime} \in G_{n-k}^{g}$ implies $Q \subset Q^{\prime}\left[\tau^{k+1}\right]$. In other words, if at the $(n-k)$ th step we made a correction 3 on the interval $Q^{\prime}$, then at the next step the correction is forbidden on the set $Q^{\prime} \backslash Q^{\prime}[\tau]$, at the $(n-k+2)$ nd step it is forbidden on the set $Q^{\prime} \backslash Q^{\prime}\left[\tau^{2}\right]$, and so on. Since $\tau / \delta \in \mathbb{N}$, an interval $Q \in$ $G_{n+1}, Q \subset Q^{\prime} \in G_{n-k}^{g}$, either lies in the set $Q^{\prime}\left[\tau^{k+1}\right]$ or does not intersect it.

\footnotetext{
${ }^{2}$ The top indices $g$ and $d$ are the first letters of "go" and "delay".

${ }^{3}$ Recall that this means that $Q^{\prime}$ belongs to the set of indices of summation in the definition (5) of the corresponding correcting term $r_{n-k-1}$.
} 
In fact, $Q^{\prime}\left[\tau^{k+1}\right] \backslash Q^{\prime}\left[\tau^{k}\right]$ are the "bad" subsets of $Q^{\prime}$ : on the $k$ th of them the "pause" lasts for $k$ steps.

We make some simple, but important, observations. It is easily seen that if $Q$ is a bounded interval and $\operatorname{dist}\left(t, c_{Q}\right)>3 \lambda \varepsilon_{n}|Q|$, then

$$
\left|F_{Q}^{\left[\varepsilon_{n}\right]}(t)\right| \leq C(\alpha) \frac{(\lambda|Q|)^{\beta}}{\left|t-c_{Q}\right|^{\beta}}
$$

for any $n \in \mathbb{N}$, and moreover,

$$
\left|\left(F_{Q}^{\left[\varepsilon_{n}\right]}\right)^{\prime}(t)\right| \leq C(\alpha) \frac{(\lambda|Q|)^{\beta}}{\left|t-c_{Q}\right|^{\beta+1}} .
$$

It follows that for all $k \in \mathbb{N}$ and all $t \in Q\left[\tau^{k}\right]$ we have

$$
\begin{gathered}
\left|F_{Q}^{\left[\varepsilon_{n}\right]}(t)\right| \leq C_{1}(\alpha) \frac{\lambda^{\beta}}{\tau^{k \beta}}, \\
\left|\left(F_{Q}^{\left[\varepsilon_{n}\right]}\right)^{\prime}(t)\right| \leq C_{1}(\alpha) \frac{\lambda^{\beta}}{|Q| \tau^{k(\beta+1)}} .
\end{gathered}
$$

Indeed, for $\tau^{k} / 2>3 \lambda \varepsilon_{n}$ these estimates coincide with the preceding ones, and for $\tau^{k} / 2>$ $3 \lambda \varepsilon_{n}$ we can use the estimates

$$
\left|F_{Q}^{\left[\varepsilon_{n}\right]}(t)\right| \leq \frac{C(\alpha)}{\varepsilon_{n}^{\beta}}
$$

and

$$
\left|\left(F_{Q}^{\left[\varepsilon_{n}\right]}\right)^{\prime}(t)\right| \leq \frac{C(\alpha)}{|Q| \lambda \varepsilon_{n}^{\beta}}, \quad t \in \mathbb{R} .
$$

We can improve the right-hand inequality in item 2) of Lemma 4 for $t \in Q\left[\tau^{k}\right]$. Indeed, applying (13) instead of (3), we get

$$
\left|h(t)-h\left(c_{Q}\right) e^{i \theta} F_{Q}^{[\varepsilon]}(t)\right| \leq \frac{C(\alpha) \lambda^{\beta}}{\tau^{k \beta}}|h(t)|, \quad t \in Q\left[\tau^{k}\right] .
$$

Let $t \in I$, and let $Q_{t}^{n}$ denote the element of $H_{n}$ determined by the condition $t \in Q_{t}^{n}$. Let $D_{n}^{k}(t):=\#\left\{l=1, \ldots, n: t \in Q_{t}^{l} \backslash Q_{t}^{l}\left[\tau^{k}\right]\right\}$. For $t \in V_{n}$, let $\widetilde{D}_{n}(t):=\#\{l=1, \ldots, n:$ $\left.Q_{t}^{l} \in G_{l}^{d}\right\}$. We know that if $Q_{t}^{l} \in G_{l}^{d}$, then for some $k_{l} \in \mathbb{N}$ we have $Q_{t}^{l} \subset Q_{t}^{l-k_{l}} \backslash$ $Q_{t}^{l-k_{l}}\left[\tau^{k_{l}}\right]$. Moreover, if $l_{1} \neq l_{2}$, then either $k_{l_{1}} \neq k_{l_{2}}$, or $l_{1}-k_{l_{1}} \neq l_{2}-k_{l_{2}}$. So, with each natural $l \leq n$ such that $Q_{t}^{l} \in G_{l}^{d}$, we can associate a pair $\left(l-k_{l}, k_{l}\right)$ such that $Q_{t}^{l} \subset Q_{t}^{l-k_{l}} \backslash$ $Q_{t}^{l-k_{l}}\left[\tau^{k_{l}}\right]$, and that mapping is injective. Hence, $\widetilde{D}_{n}(t) \leq \sum_{k \in \mathbb{N}} D_{n}^{k}(t)=: D_{n}(t)$. The next section is devoted to estimating the lengths of the sets $E_{n}:=\left\{t \in I: D_{n}(t) \geq n / 2\right\}$.

\section{§6. Estimates FOR THE LENGTHS OF THE SETS $E_{n}$}

Lemma 5. For $\tau$ sufficiently small, we have

$$
\left|E_{n}\right| \leq \frac{C(\tau)}{n^{2}}
$$

where $C(\tau)$ tends to zero as $\tau \rightarrow 0$.

Proof. Let

$$
\xi_{i}^{(k)}:=\sum_{Q \in H_{i}} \chi_{Q \backslash Q\left[\tau^{k}\right]}, \quad \xi_{i}^{(k)}:={\xi^{\prime}}_{i}^{(k)}-\tau^{k}
$$


Then, viewing $\xi_{i}^{(k)}$ as random variables on the probability space $I$ with the measure $d x$, we have $E \xi_{i}^{(k)}=0$. We can describe the sets $E_{n}$ in terms of $\xi_{i}^{(k)}$ as follows:

$$
x \in E_{n} \Longleftrightarrow \sum_{k=1}^{n-1} \sum_{i=1}^{n-k} \xi_{i}^{(k)}(x) \geq \frac{n}{2} .
$$

Thus, we need to estimate the probabilities of the event that the sums $\sum_{i=1}^{n-k} \xi_{i}^{\prime(k)}$ of random variables are large. It is easily seen that the random variables $\xi_{i}^{(1)}, i=1,2, \ldots$, are independent: this follows from the fact that $\tau / \delta$ is an integer. Unfortunately, for $k>1$ we cannot say the same about $\xi_{i}^{(k)}, i=1,2, \ldots$, because $\tau^{k} / \delta$ may fail to be an integer. But for $k>1$ the variables $\xi_{i}^{\prime(k)}, i=1,2, \ldots$, are still "almost independent" in a sense, and we shall use that.

Observe that if $j \geq i+k$, then $\xi_{i}^{(k)}$ is constant on each interval in $H_{j}$ (because $\left.\tau^{k} / \delta^{k} \in \mathbb{N}\right)$. Consequently, the following is true:

1) if $i_{1}+k \leq i_{2} \leq i_{3} \leq i_{4}$, then $E\left(\xi_{i_{1}}^{(k)} \xi_{i_{2}}^{(k)} \xi_{i_{3}}^{(k)} \xi_{i_{4}}^{(k)}\right)=0$ (because the function $\xi_{i_{2}}^{(k)} \xi_{i_{3}}^{(k)} \xi_{i_{4}}^{(k)}$ is periodic with period $\delta^{i_{1}+k}$, and $\xi_{i_{1}}^{(k)}$ is constant on each interval in $\left.H_{i_{1}+k}\right)$;

$2)$ if $i_{1} \leq i_{2} \leq i_{3} \leq i_{4}-k$, then $E\left(\xi_{i_{1}}^{(k)} \xi_{i_{2}}^{(k)} \xi_{i_{3}}^{(k)} \xi_{i_{4}}^{(k)}\right)=0$ (because the function $\xi_{i_{4}}^{(k)}$ is periodic with period $\delta^{i_{3}+k}$, and $\xi_{i_{1}}^{(k)} \xi_{i_{2}}^{(k)} \xi_{i_{3}}^{(k)}$ is constant on each interval in $H_{i_{3}+k}$ ).

Now we write

$$
\begin{aligned}
P\left(\left|\sum_{i=1}^{n} \xi_{i}^{(k)}\right|>\varepsilon\right) & \leq \frac{E\left(\xi_{1}^{(k)}+\cdots+\xi_{n}^{(k)}\right)^{4}}{\varepsilon^{4}} \\
& =\frac{\sum_{\left(i_{1}, i_{2}, i_{3}, i_{4}\right) \in\{1, \ldots, n\}^{4}} E\left(\xi_{i_{1}}^{(k)} \xi_{i_{2}}^{(k)} \xi_{i_{3}}^{(k)} \xi_{i_{4}}^{(k)}\right)}{\varepsilon^{4}}
\end{aligned}
$$

First, note that $E\left(\xi_{i_{1}}^{(k)} \xi_{i_{2}}^{(k)} \xi_{i_{3}}^{(k)} \xi_{i_{4}}^{(k)}\right) \leq E\left|\xi_{i_{1}}^{(k)}\right|=2 \tau^{k}\left(1-\tau^{k}\right) \leq 2 \tau^{k}$ (the first inequality is true because $\left|\xi_{i}^{(k)}\right|<1$ for all $i$ and $\left.k\right)$. Second, if $\left(j_{1}, j_{2}, j_{3}, j_{4}\right)$ is a monotone nondecreasing permutation of the numbers $\left(i_{1}, i_{2}, i_{3}, i_{4}\right)$, then a term of the form $E\left(\xi_{i_{1}}^{(k)} \xi_{i_{2}}^{(k)} \xi_{i_{3}}^{(k)} \xi_{i_{4}}^{(k)}\right)$ may differ from zero only if $j_{2}-j_{1}<k$ and $j_{4}-j_{3}<k$ (by the above observation). But the number of such quads $\left(j_{1}, j_{2}, j_{3}, j_{4}\right)$ does not exceed $\frac{n(n-1)}{2} k^{2}$. Hence, the number of nonzero terms in the numerator in (17) is at most $4 ! \frac{n(n-1)}{2} k^{2}$. Therefore,

$$
P\left(\left|\sum_{i=1}^{n} \xi_{i}^{(k)}\right|>\varepsilon\right) \leq \frac{C n^{2} k^{2} \tau^{k}}{\varepsilon^{4}} .
$$

Let $E_{n}^{k}:=\left\{t \in I: D_{n}^{k}(t) \geq n\left(\sqrt[8]{(4 \tau)^{k}}+\tau^{k}\right)\right\}$. If $\tau$ is so small that $\sum_{1}^{\infty} \sqrt[8]{(4 \tau)^{k}}+\tau^{k}<$ $\frac{1}{2}$, then $\left|E_{n}\right| \leq \sum_{k=0}^{\infty}\left|E_{n}^{k}\right|$. Finally, since

$$
D_{n}^{k}(t)=\sum_{i=1}^{n} \xi_{i}^{(k)}(t)=\sum_{i=1}^{n} \xi_{i}^{(k)}(t)+n \tau^{k},
$$

we can use (18) to get

$$
\left|E_{n}\right| \leq \sum_{k=1}^{\infty}\left|E_{n}^{k}\right| \leq \sum_{k=1}^{\infty} \frac{C k^{2} \tau^{\frac{k}{2}}}{n^{2} 2^{k}}
$$

Lemma 5 follows from this estimate. 


\section{$\S 7$. Estimates of $f_{n}$}

In this section, we prove some estimates for the functions $f_{n}$, of which the following two are principal: estimate (24), which allows us to apply Lemma 4, and estimate (20), which shows that for $\lambda$ sufficiently small the terms $f_{n}\left(c_{Q^{\prime}}\right) F_{Q^{\prime}}^{\left[\varepsilon_{n}\right]} e^{i \theta}$ corresponding to $Q^{\prime} \neq Q$ do not change the situation on $Q$ essentially. All the estimates we prove do not depend on the choice of $G_{n}$, but depend on how we choose the subsets $G_{n}^{g}$ (namely, we use estimates (13) and (14)). As was mentioned above, the sets $G_{n}$ will be defined later. We put

$$
T_{n+1}(t):=\sum_{Q \in G_{n+1}^{g}, Q \neq Q_{t}^{n+1}} f_{n}\left(c_{Q}\right) F_{Q}^{\left[\varepsilon_{n}\right]} e^{i \theta} .
$$

Recall that the interval $Q_{t}^{n+1} \in H_{n+1}$ is determined by the condition $t \in Q_{t}^{n+1}$.

Lemma 6. There exists a positive number $\rho=\rho(\alpha)$ such that if $\lambda>0$ is sufficiently small and $\delta=\delta(\lambda)>0$ is sufficiently small, then the following are true:

1) for all $t \in I$,

$$
\left|T_{n+1}(t)\right| \leq \frac{c(\alpha) \lambda^{\beta}\left|f_{n}(t)\right|}{\rho} ;
$$

2) if $k \leq n, x \in V_{n+1}^{g}, y \in I$, and $|x-y| \leq \delta^{k}$, then

$$
\left|f_{n}(x)\right| \leq \frac{\left|f_{n}(y)\right|}{\rho^{n-k+1}}
$$

3) for all $t \in I$,

$$
\left|T_{n+1}^{\prime}(t)\right| \leq \frac{c(\alpha) \lambda^{\beta}\left|f_{n}(t)\right|}{\delta^{n+1} \rho}
$$

4) for all $t \in V_{n+1}^{g}$,

$$
\left|f_{n}^{\prime}(t)\right| \leq \frac{c_{1}(\alpha) \lambda^{\beta}\left|f_{n}(t)\right|}{\delta^{n}}
$$

5) for all $Q \in G_{n+1}^{g}$,

$$
\operatorname{osc}_{Q} f_{n} \leq \kappa\left|f_{n}\left(c_{Q}\right)\right| .
$$

Proof. Inequality (24) clearly follows from (23) if we take $\delta$ sufficiently small (the interval $Q$ in (24) has length $\delta^{n+1}$ ). We derive (20) and (22) from (21), which, in its turn, follows from (20) and (24) for the preceding $n$. Finally, (23) follows from (24) and (22) for the preceding $n$.

The base of induction, that is, (24) and (21) for $n=1$, is provided by the condition $f_{1}(t) \neq 0, t \in I$ (see $\left.\S 3\right)$ and by the choice of sufficiently small $\rho$ and $\delta$.

We deduce (20) and (22) from (21). Fix $t \in I$. Let $G_{\epsilon}$ be the set of all intervals $Q^{\prime} \in H_{n+1}$ with $\operatorname{dist}\left(c_{Q^{\prime}}, Q_{t}^{n+1}\right) \geq \epsilon$. We denote

$$
\sigma_{\epsilon}(t):=\sum_{Q^{\prime} \in G_{\epsilon}}\left|F_{Q^{\prime}}^{\left[\varepsilon_{n}\right]}(t)\right|, \quad \sigma_{\epsilon}^{*}(t):=\sum_{Q^{\prime} \in G_{\epsilon}}\left|\left(F_{Q^{\prime}}^{\left[\varepsilon_{n}\right]}\right)^{\prime}(t)\right| .
$$

We shall need the estimates

$$
\sigma_{\epsilon}(t) \leq c(\alpha) \lambda^{\beta}\left(\frac{\delta^{n+1}}{\epsilon}\right)^{\alpha}
$$

and

$$
\sigma_{\epsilon}^{*}(t) \leq c(\alpha) \lambda^{\beta} \frac{\delta^{(n+1) \beta}}{\epsilon^{\beta+1}} .
$$


They can be obtained by termwise estimation with the help of (11) and (12), respectively, followed by estimating the sum by an integral. A detailed proof of (25) can be found in [2, page 234], and (26) can be proved similarly.

In order to get the upper estimates of $|T|$ and $\left|T^{\prime}\right|$, we shall split the terms on the right-hand side of (19) into several groups, in accordance with their distance from the point $t$. For each group, we estimate $\left|f\left(c_{Q}\right)\right|$ with the help of (21) (the closer to $t$ is the interval $Q$, the better is this estimate), and then apply (25) (respectively, (26) for $\left|T^{\prime}\right|$ ), which, by contrast, becomes better when $\epsilon$ grows.

So, let $G_{n+1}^{g}:=\bigsqcup_{k<n+1} G^{[k]}$, where $G^{[n+1]}:=G_{n+1}^{g} \backslash G_{\delta^{n}}, G^{[k]}:=\left(G_{n+1}^{g} \cup G_{\delta^{k}}\right) \backslash G_{\delta^{k-1}}$. For $y \in G^{[k]}$ we have $\left|f_{n}(y)\right| \leq\left|f_{n}(t)\right| / \rho^{n-k+2}$ and $\operatorname{dist}\left(G^{[k]}, t\right) \geq \delta^{k} / 2$, whence

$$
\begin{aligned}
\left|T_{n+1}(t)\right| & \leq \sum_{k} \sum_{Q^{\prime} \in G^{[k]}}\left|F_{Q^{\prime}}^{\left[\varepsilon_{n}\right]}(t)\right|\left|f_{n}(t)\right| / \rho^{n-k+2} \\
& \leq c(\alpha) \lambda^{\beta}\left|f_{n}(t)\right| \sum_{k} \frac{\delta^{(n+1) \beta}}{\delta^{k \beta} \rho^{n-k+2}} \leq c(\alpha) \lambda^{\beta}\left|f_{n}(t)\right| \rho^{-1} \sum_{k}\left(\frac{\delta^{\beta}}{\rho}\right)^{n-k+1} .
\end{aligned}
$$

Similarly,

$$
\begin{aligned}
\left|T_{n+1}^{\prime}(t)\right| & \leq \sum_{k} \sum_{Q^{\prime} \in G^{[k]}}\left|\left(F_{Q^{\prime}}^{\left[\varepsilon_{n}\right]}\right)^{\prime}(t)\right|\left|f_{n}(t)\right| / \rho^{n-k+2} \\
& \leq c(\alpha) \frac{\lambda^{\beta}\left|f_{n}(t)\right|}{\delta^{n+1}} \sum_{k} \frac{\delta^{(n+1)(\beta+1)}}{\delta^{k \beta+1} \rho^{n-k+2}} \\
& \leq c(\alpha) \frac{\lambda^{\beta}\left|f_{n}(t)\right|}{\delta^{n+1} \rho} \sum_{k}\left(\frac{\delta^{\beta+1}}{\rho}\right)^{n-k+1}
\end{aligned}
$$

Choosing $\delta$ so that $\frac{\delta^{\beta}}{\rho}<\frac{1}{2}$, we get (20) and (22).

Now we prove that (21) and (23) follow from (20), (22), and (24) for the smaller $n$. We need the estimate

$$
\left|f_{n}(x)\right| \leq \frac{4}{\theta}\left|f_{n+1}(x)\right| \leq \cdots \leq\left(\frac{4}{\theta}\right)^{k}\left|f_{n+k}(x)\right|, \quad x \in I, n, k \in \mathbb{N},
$$

which is true for $\lambda$ sufficiently small. Let us prove this. Let $Q_{x}^{n+1} \in G_{n+1}^{g}$. Then

$$
\begin{aligned}
\left|f_{n}(x)\right| & \leq \frac{2}{\theta}\left|f_{n}(x)-f_{n}\left(c_{Q_{x}}\right) e^{i \theta} F_{Q_{x}}^{\left[\varepsilon_{n}\right]}(x)\right| \\
& \leq \frac{2}{\theta}\left(\left|f_{n+1}(x)\right|+\left|T_{n+1}(x)\right|\right) \leq \frac{2}{\theta}\left(\left|f_{n+1}(x)\right|+c(\alpha) \lambda^{\beta} \rho^{-1}\left|f_{n}(x)\right|\right) .
\end{aligned}
$$

The first inequality follows from item 2) of Lemma 4 (which is applicable because of (24)); the last inequality is a consequence of (20). The inequality $\left|f_{n}(x)\right| \leq \frac{4}{\theta}\left|f_{n+1}(x)\right|$ follows from the above estimate if $2 c(\alpha) \lambda^{\beta} \theta^{-1} \rho^{-1}<\frac{1}{2}$. If $Q_{x}^{n+1} \notin G_{n+1}^{g}$, then the same inequality follows from (20) even easier. Thus, (27) is proved.

Now, suppose $k \leq n+1, x \in V_{n+2}^{g}, y \in I$, and $|x-y| \leq \delta^{k}$. Let $k^{\prime}:=\max \{l \leq n$ : $\left.x \in V_{l+1}^{g}\right\}$. The fact that $x$ is again in a "good" set, namely, in $V_{n+2}^{g}$, implies that

$$
x \in Q_{x}^{k^{\prime}+1}\left[\tau^{n-k^{\prime}+1}\right] .
$$


First, assume that $k \leq k^{\prime}$. Then

$$
\begin{aligned}
\left|f_{n+1}(x)\right| & =\left|f_{n} x\right|+\left|T_{n+1}(x)\right| \leq\left|f_{n}(x)\right|\left(1+c(\alpha) \frac{\lambda^{\beta}}{\rho}\right) \leq \cdots \\
& \leq\left|f_{k^{\prime}+1}(x)\right|\left(1+c(\alpha) \frac{\lambda^{\beta}}{\rho}\right)^{n-k^{\prime}} \\
& \leq\left(\left|f_{k^{\prime}}(x)\right|+\left|T_{k^{\prime}+1}(x)\right|+\left|f_{k^{\prime}}\left(c_{Q_{x}^{k^{\prime}}}\right)\right|\left|F_{Q_{x}^{k^{\prime}+1}}^{\left[\varepsilon_{n}\right]}\right|\right)\left(1+c(\alpha) \frac{\lambda^{\beta}}{\rho}\right)^{n-k^{\prime}} \\
& \leq\left|f_{k^{\prime}}(x)\right|\left(1+c(\alpha) \frac{\lambda^{\beta}}{\rho}+(1+\kappa)\left|F_{Q_{x}^{k^{\prime}+1}}^{\left[\varepsilon_{n}\right]}\right|\right)\left(1+c(\alpha) \frac{\lambda^{\beta}}{\rho}\right)^{n-k^{\prime}} .
\end{aligned}
$$

Applying (13) and taking (28) into account, we continue:

$$
\begin{aligned}
\left|f_{n+1}(x)\right| & \leq\left|f_{k^{\prime}}(x)\right|\left(1+C(\alpha) \lambda^{\beta}\left(\frac{1}{\rho}+\frac{(1+\kappa)}{\tau^{\left(n-k^{\prime}+1\right) \beta}}\right)\right)\left(1+c(\alpha) \frac{\lambda^{\beta}}{\rho}\right)^{n-k^{\prime}} \\
& \leq \frac{\left|f_{k^{\prime}}(y)\right|}{\rho^{k^{\prime}-k+1}}\left(1+C(\alpha) \lambda^{\beta}\left(\frac{1}{\rho}+\frac{(1+\kappa)}{\tau^{\left(n-k^{\prime}+1\right) \beta}}\right)\right)\left(1+c(\alpha) \frac{\lambda^{\beta}}{\rho}\right)^{n-k^{\prime}} \\
& \leq\left|f_{n+1}(y)\right| \frac{\left(\frac{4}{\theta}\right)^{n-k^{\prime}+1}}{\rho^{k^{\prime}-k+1}}\left(1+C(\alpha) \lambda^{\beta}\left(\frac{1}{\rho}+\frac{(1+\kappa)}{\tau^{\left(n-k^{\prime}+1\right) \beta}}\right)\right)\left(1+c(\alpha) \frac{\lambda^{\beta}}{\rho}\right)^{n-k^{\prime}} .
\end{aligned}
$$

We have used the inductive hypothesis (21) for $n=k^{\prime}$ and (27). Now, choosing $\rho$ so that $\sqrt{\rho}<\theta / 50$ and $\sqrt{\rho}<\tau^{\beta} / 50(C(\alpha))$, we get

$$
\begin{aligned}
\left|f_{n+1}(x)\right| \leq & \left|f_{n+1}(y)\right| \frac{1}{\rho^{k^{\prime}-k+1}} 5^{-n-k^{\prime}+1} \rho^{-n+k^{\prime}-1} \\
& \times\left(\left(1+c(\alpha) \frac{\lambda^{\beta}}{\rho}\right)^{n-k^{\prime}+1}+5^{-n-k^{\prime}+1} \rho^{-n+k^{\prime}-1}\left(1+c(\alpha) \frac{\lambda^{\beta}}{\rho}\right)^{n-k^{\prime}}\right) .
\end{aligned}
$$

Then we choose $\lambda=\lambda(\rho)$ so that $\left(1+c(\alpha) \frac{\lambda^{\beta}}{\rho}\right)<2$, obtaining

$$
\left|f_{n+1}(x)\right| \leq \frac{\left|f_{n+1}(y)\right|}{\rho^{n-k^{\prime}+1+k^{\prime}-k+1}}
$$

and we are done.

Now we prove (23). Let $t \in V_{n+2}^{g}$. As in the proof of (21), let $k^{\prime}:=\max \{l \leq n$ : $\left.x \in V_{l+1}^{g}\right\}$. Again, (28) is fulfilled. We have

$$
\begin{aligned}
\left|\left(f_{n+1}\right)^{\prime}(t)\right| & \leq\left|f_{n}^{\prime}(t)\right|+\left|T_{n+1}^{\prime}(t)\right| \leq \cdots \\
& \leq\left|f_{k^{\prime}}^{\prime}(t)\right|+\left|f_{k^{\prime}}\left(c_{Q_{t}^{k^{\prime}+1}}\right)\right|\left|\left(F_{Q_{t}^{k^{\prime}+1}}^{\left[\varepsilon_{k^{\prime}}\right]}\right)^{\prime}(t)\right|+\sum_{l=k^{\prime}+1}^{n+1}\left|T_{l}^{\prime}(t)\right| .
\end{aligned}
$$

Applying the inductive hypothesis ((23) for $n=k^{\prime}$ ) and (27), we obtain

$$
\left|f_{k^{\prime}}^{\prime}(t)\right| \leq c_{1}(\alpha) \lambda^{\beta} \frac{\left|f_{k^{\prime}}(t)\right|}{\delta^{k^{\prime}}} \leq c_{1}(\alpha) \lambda^{\beta}(4 / \theta)^{n-k^{\prime}+1} \frac{\left|f_{n+1}(t)\right|}{\delta^{k^{\prime}}} .
$$

To estimate the second term in (29), we use (24), then (28) and (14), and finally (27), in the following way:

$$
\begin{aligned}
\left|f_{k^{\prime}}\left(c_{Q_{t}^{k^{\prime}+1}}\right) \|\left(F_{Q_{t}^{k^{\prime}+1}}^{\left[\varepsilon_{k^{\prime}}\right]}\right)^{\prime}(t)\right| & \leq(1+\kappa)\left|f_{k^{\prime}}(t)\right| \frac{c(\alpha) \lambda^{\beta}}{\delta^{k^{\prime}+1} \tau^{\beta\left(n-k^{\prime}+1\right)}} \\
& \leq(1+\kappa)(4 / \theta)^{n-k^{\prime}+1}\left|f_{n+1}(t)\right| \frac{c(\alpha) \lambda^{\beta}}{\delta^{k^{\prime}+1} \tau^{\beta\left(n-k^{\prime}+1\right)}} .
\end{aligned}
$$


Finally, using (22) and then (27) once again, we see that

$$
\left|T_{l+1}^{\prime}(t)\right| \leq \frac{c(\alpha) \lambda^{\beta}\left|f_{l}(t)\right|}{\rho \delta^{l+1}} \leq(4 / \theta)^{n+1-l} \frac{c(\alpha) \lambda^{\beta}\left|f_{n+1}(t)\right|}{\rho \delta^{l+1}} .
$$

Taking $\delta<\theta \tau^{\beta} /(4 A)$ and substituting all these estimates in (29), we get

$$
\left|f_{n+1}^{\prime}(t)\right| \leq \frac{\lambda^{\beta}\left|f_{n+1}(t)\right|}{\delta^{n+1}}\left(c_{1}(\alpha) / A^{n-k^{\prime}+1}+c(\alpha) \frac{4(1+\kappa)}{\theta \tau^{\beta}}+\frac{4}{\rho \theta} \sum_{k=0}^{n-k^{\prime}+1} A^{-k}\right) .
$$

Taking $A>2$, we arrive at (23), provided $c_{1}(\alpha)$ is sufficiently large.

\section{§. END OF THE PROOF}

In order to complete the construction, we must define the sets $G_{n}$. We fix the parameter $\tau$ so as to satisfy the inequality $C(\tau) \sum_{n=1}^{\infty} \frac{1}{n^{2}}<\frac{1}{8}$, where $C(\tau)$ is the constant occurring in Lemma 5.

We define the sets $G_{n+1}$ as follows: $G_{1}:=H_{1}=\{I\}$; an interval $Q \in H_{n+1}$ belongs to $G_{n+1}$ if $Q \subset V_{n}$,

$$
M_{Q}\left(f_{n}\right) \leq K_{n} \eta^{n}
$$

with $K_{n}$ and $\eta$ to be defined later, and

$$
\widetilde{D_{n}}\left(c_{Q}\right) \leq n / 2
$$

(in fact, of course, $\widetilde{D_{n}}$ is a constant on $Q$ ). Note that the choice of $\tau$ and Lemma 5 guarantee that the total (over all $n$ ) length of the intervals $Q \in H_{n+1}, Q \subset V_{n}$, not included in $G_{n+1}$ because of a violation of condition (31), does not exceed $\frac{1}{8}$.

Lemma 7. There exists a constant $C^{\prime}(\alpha)$ such that for all sufficiently small $\lambda$ the following inequalities are valid:

1) if $Q \in G_{n+1}^{g}$, then

$$
\int_{Q}\left|f_{n+1}\right|^{p} \leq X \int_{Q}\left|f_{n}\right|^{p}
$$

2) if $Q \in G_{n+1}^{d}$, then

$$
\int_{Q}\left|f_{n+1}\right|^{p} \leq Y \int_{Q}\left|f_{n}\right|^{p}
$$

where $X:=\gamma(\lambda)^{p}\left(1+C^{\prime}(\alpha) \lambda^{\beta}\right)^{p}\left(=(1+B \lambda)\left(1+C^{\prime}(\alpha) \lambda^{\beta}\right)^{p}\right), Y:=\left(1+C^{\prime}(\alpha) \lambda^{\beta}\right)^{p}$.

Proof. We put $P_{n+1}(t):=f_{n}(t)-f_{n}\left(c_{Q_{t}}\right) e^{i \theta} F_{Q_{t}}^{[\varepsilon]}(t)$; then for $Q \in G_{n+1}^{g}$ we get

$$
\int_{Q}\left|f_{n+1}\right|^{p}=\int_{Q}\left|P_{n+1}+T_{n+1}\right|^{p} \leq \int_{Q}\left|P_{n+1}\right|^{p}\left(1+\frac{\left|T_{n+1}\right|}{\left|P_{n+1}\right|}\right)^{p} .
$$

Applying statement 2) of Lemma 4 and estimate (20), we see that

$$
\int_{Q}\left|f_{n+1}\right|^{p} \leq \int_{Q}\left|P_{n+1}\right|^{p}\left(1+\frac{2 c(\alpha) \lambda^{\beta}}{\rho \theta}\right)^{p} .
$$

Now, estimating the integral by using statement 1) of Lemma 4 , we obtain

$$
\int_{Q}\left|f_{n+1}\right|^{p} \leq(1+B \lambda)\left(1+C^{\prime}(\alpha) \lambda^{\beta}\right)^{p} \int_{Q}\left|f_{n}\right|^{p} .
$$

Recall that the applicability of Lemma 4 is ensured by (24).

The second case is even easier and is left to the reader. 
Lemma 8. If $\lambda$ is sufficiently small, then for all $n$ we have

$$
\int_{V_{n}}\left|f_{n}\right|^{p} \leq \eta^{n} \int_{V_{1}}\left|f_{1}\right|^{p}
$$

with some $\eta \in(0,1)$.

Proof. Denote by $\Theta_{n}$ the set $\{0,1\}^{n}$ (the set of all ordered $n$-tuples $v=\left(v_{1}, \ldots, v_{n}\right)$ of zeros and units). For $v \in \Theta_{n}$, we define a set $G^{(v)} \subset G_{n}$ as the set of all $Q$ 's such that, for all $k, v_{k}=1$ if and only if $Q \subset V_{k}^{g}$. For $v \in \Theta_{n}$, put $Z(v):=\prod_{k=1}^{n} X^{v_{k}} Y^{\left(1-v_{k}\right)}$. We define $G_{1}^{g}:=I$ and use induction on $n$ to prove the estimate

$$
\int_{I}\left|f_{1}\right|^{p} \geq X \sum_{v \in \Theta_{n}} Z(v)^{-1} \int_{G^{(v)}}\left|f_{n}\right|^{p}
$$

The base of induction is obvious. Suppose (33) is true for some $n$. Then

$$
\begin{aligned}
Z(v)^{-1} \int_{G^{(v)}}\left|f_{n}\right|^{p} & =Z(v)^{-1}\left(\int_{G^{(v, 1)}}\left|f_{n}\right|^{p}+\int_{G^{(v, 0)}}\left|f_{n}\right|^{p}\right) \\
& \geq Z(v)^{-1}\left(X^{-1} \int_{G^{(v, 1)}}\left|f_{n+1}\right|^{p}+Y^{-1} \int_{G^{(v, 0)}}\left|f_{n+1}\right|^{p}\right) \\
& =Z(v, 1)^{-1} \int_{G^{(v, 1)}}\left|f_{n+1}\right|^{p}+Z(v, 0)^{-1} \int_{G^{(v, 0)}}\left|f_{n+1}\right|^{p}
\end{aligned}
$$

(we have used Lemma 7). This proves the inductive step. Next, for $\lambda$ sufficiently small we have $X<1$ and $Y>1$. If $n>N_{0}$ and $v \in \Theta_{n}$, then $\sum_{k} v_{k}<n / 2$ implies $G^{(v)}=\varnothing$ (this follows from (31)); therefore, the right-hand side in (33) is not less than $X^{-\frac{n}{2}+1} Y^{-\frac{n}{2}} \int_{V_{n}}\left|f_{n}\right|^{p}$, and (32) follows provided $\lambda$ is sufficiently small.

Now we are ready to finish the description of our construction. What we should do is to make condition (30) precise. We take $\eta$ as in Lemma 8. Since $\int_{V_{n}}\left|f_{n}\right|^{p} \leq C \eta^{n}$ by Lemma 8, the total length of all intervals $Q \in H_{n+1}$ such that $Q \subset V_{n}$ and condition (30) fails on $Q$ does not exceed $\frac{C}{K_{n}}$. For the role of $K_{n}$ we take a sequence growing as some power of $n$ and satisfying the condition $\sum_{n=1}^{\infty} \frac{C}{K_{n}}<\frac{1}{8}$. Then the total length of all intervals removed from $V_{n}$ at all steps because of a violation of (30) is less than $\frac{1}{8}$. But the same can be said about the length of all intervals removed because of a violation of (31). Hence, $\left|\bigcap_{i=1}^{\infty} V_{n}\right|>\frac{3}{4}$.

Condition (30) and estimate (24) of the oscillation of $f_{n}$ show that if $Q \in G_{n+1}$, then $\left|f_{n}\left(c_{Q}\right)\right| \leq C^{\prime} \eta^{\prime n}$. Consequently, taking (3) and (25) into account, we get

$$
\left|f_{n}(t)-f_{n+1}(t)\right| \leq \frac{C_{1} \eta^{\prime n}}{\varepsilon_{n}^{\beta}}+C_{2} \lambda^{\beta} \eta^{\prime n}, \quad t \in \mathbb{R} .
$$

The first term on the right-hand side corresponds to the building block $F_{Q_{t}^{n+1}}^{\left[\varepsilon_{n}\right]}$ (if there is any), and the second corresponds to all others. This estimate implies that the $f_{n}$ converge uniformly on $\mathbb{R}$ to some function $f$. We also need the estimate

$$
\left|f_{n}(t)-f_{n+1}(t)\right| \leq c \frac{\lambda^{\beta} \delta_{n+1}^{\alpha} C^{\prime} \eta^{\prime n}}{|t|^{\beta}}, \quad t \notin 3 I,
$$

which follows from (111). Now, (35) implies that

$$
\left|f_{n}(t)\right| \leq c|t|^{-\beta}, \quad|t| \geq 3 / 2
$$

so that, fixing $t$, we can write

$$
\int_{\mathbb{R}} \frac{f_{n}(s) d s}{|s-t|^{1-\alpha}}=\int_{|s| \leq \max (2|t|, 3 / 2)} \frac{f_{n}(s) d s}{|s-t|^{1-\alpha}}+\int_{|s| \geq \max (2|t|, 3 / 2)} \frac{f_{n}(s) d s}{|s-t|^{1-\alpha}} .
$$


In the first term, passage to the limit in the integral is justified by the uniform convergence of $f_{n}$ as $n \rightarrow \infty$; in the second, the integrand is majorized by $c|s|^{-2}$. Consequently,

$$
\lim _{n \rightarrow \infty} g_{n}=\lim _{n \rightarrow \infty} \int_{\mathbb{R}} \frac{f_{n}(s)}{|s-t|^{1-\alpha}}=\int_{\mathbb{R}} \frac{f(s)}{|s-t|^{1-\alpha}} .
$$

(The first identity follows from Lemma 1.) It remains to observe that the functions $g_{n}$ converge uniformly to $g$ :

$$
\left|r_{n}\right| \leq \frac{C^{\prime \prime} \eta^{\prime n}}{\varepsilon_{n}}
$$

So, the program announced at the beginning of $\S 3$ is complete. Now we show that the function $f$ satisfies Hölder's condition.

In this argument, the constants may depend on all parameters except for $n$. Of course, we use the proposition mentioned at the beginning of $\S 4$. The condition $\left|f_{n}-f\right| \leq C_{1} \eta_{1}^{n}$ follows easily from (34).

We estimate the derivative of the correction term at the $n$th step:

$$
\begin{aligned}
\left|\left(W_{\alpha} r_{n}\right)^{\prime}(t)\right| & =\left|\frac{d}{d t}\left(\sum_{Q \in G_{n+1}^{g}} f_{n}\left(c_{Q}\right) F_{Q}^{\left[\varepsilon_{n}\right]}(t) e^{i \theta}\right)\right| \\
& \leq \max _{Q \in G_{n+1}^{g}}\left|f_{n}\left(c_{Q}\right)\right| \sum_{Q \in G_{n+1}^{g}}\left|\left(F_{Q}^{\left[\varepsilon_{n}\right]}(t)\right)^{\prime}\right| \\
& \leq \max _{Q \in G_{n+1}^{g}}\left|f_{n}\left(c_{Q}\right)\right|\left(\left|\left(F_{Q_{t}^{n+1}}^{\left[\varepsilon_{n}\right]}(t)\right)^{\prime}\right|+\sum_{\substack{Q \in H_{n+1} \\
Q \neq Q_{t}^{n+1}}}\left|\left(F_{Q}^{\left[\varepsilon_{n}\right]}(t)\right)^{\prime}\right|\right) .
\end{aligned}
$$

We know that $\max _{Q \in G_{n+1}^{g}}\left|f_{n}\left(c_{Q}\right)\right| \leq C^{\prime} \eta^{\prime n}$. For the first term in brackets we use (8), and for the sum we use (26) with $\epsilon:=\frac{\delta^{n+1}}{2}$. Thus, we have

$$
\left|\left(W_{\alpha} r_{n}\right)^{\prime}(t)\right| \leq C^{\prime} \eta^{\prime n}\left(\frac{C_{1}}{\delta^{n+1} \varepsilon_{n}}+\frac{C_{2}}{\delta^{n+1}}\right) \leq \frac{C_{3}}{\delta^{\prime n+1}} .
$$

Hence, $\left|f_{n}^{\prime}(t)\right| \leq \frac{C_{3}}{1-\delta^{\prime}} \delta^{\prime-n-1} \leq \frac{C_{4}}{\delta^{\prime n+1}}$, and Hölder's property for $f$ is proved.

\section{§9. Remark on the ORDER OF CHOOSING the PARAMETERS}

We recall the order in which we chose our parameters. Given $\alpha \in(0,1)$, we fix $p$ and $\theta$ (Lemmas 3 and 4 ). Here Lemma 4 applies for all sufficiently small $\lambda$ and $\varepsilon$. Then we fix a sequence $\varepsilon_{n}$. Independently of the other parameters, we fix $\tau$. Lemma 6 is valid for all sufficiently small $\lambda$ and $\delta$, independently of the choice of $G_{n}$ (here the smallness of $\lambda$ and $\delta$ should depend on the quantity $\rho$ occurring in that lemma, and $\rho$ itself only depends on $\alpha, \theta, p, \tau$, and the choice of the initial function $\left.g_{1}\right)$. Now we choose $\lambda$ such that the factor $\eta$ of the integral on the right in (32) is less than 1 (reducing $\lambda$ kills the influence of the "tails" $T_{n}$ in comparison with the correction effect provided by statement 1) of Lemma 2 ; note that the latter effect also drops as $\lambda$ reduces $(\gamma(\lambda) \stackrel{\lambda \rightarrow 0}{\longrightarrow} 1$ !), but the "tails" die faster). Finally, we fix $\delta$ so as to ensure (24).

So far, our considerations did not depend on $G_{n}$, so that we had no need to define these sets. Now, we fix a sequence $K_{n}$, thus finishing the description of our construction.

\section{$\S 10$. The CASE OF Negative $\alpha$}

It is natural to ask whether there is an analog of Theorem 1 for other Rietz kernels. In the case of the kernel $|x|^{-\beta}, \quad 1<\beta<2$, the answer is in the positive; moreover, since the convolution with such a kernel is, at least formally, the inverse operator to $U_{\beta-1}$ (see 
Lemma 1, statements 2) and 5)), this example coincides in essence with that constructed above.

Theorem 2. There exists a nonzero continuous function $g: \mathbb{R} \rightarrow \mathbb{C}$ with supp $g \subseteq 3 I$ and a set $E$ of positive measure such that $t \in E \Rightarrow g(t)=0, \int_{\mathbb{R}} g(x)|t-x|^{-\beta} d x=0$. The last integral converges absolutely for every $t \in E$. The function $g$ satisfies Hölder's condition with the exponent $\beta-1$.

Proof. For the role of $g$ we take the function constructed in Theorem 1 (with $\beta-1$ in place of $\alpha$ ). Let

$$
E:=V \cap(I \backslash S), \quad \text { where } S:=\bigcup_{n \in \mathbb{N}} \bigcup_{Q \in G_{n+1}^{g}} 3 \varepsilon_{n}\left(Q-c_{Q}\right)+c_{Q} .
$$

The idea is that $|S|<\frac{3}{4}$, but now supp $g_{n}$ is contained "deep" inside $S$ :

$$
\operatorname{dist}\left(\operatorname{supp} g_{n}, E\right) \geq \varepsilon_{n} \delta^{n+1} \text {. }
$$

We know (see statement 5) of Lemma 1) that

$$
f_{n}(t)=\int_{\mathbb{R}} g_{n}(x)|t-x|^{-\beta} d x \stackrel{n \rightarrow \infty}{\longrightarrow} 0
$$

for $t \in E$. To prove Theorem 2, it suffices to justify passage to the limit in the integral. The role of an integrable majorant is played by the function

$$
\widetilde{g}(x):=|t-x|^{-\beta} \sum_{n=1}^{\infty}\left|g_{n+1}(x)-g_{n}(x)\right| .
$$

We estimate the $n$th term:

$$
\left|g_{n+1}-g_{n}\right| \leq \sum_{Q \in G_{n+1}^{g}}\left|f_{n}\left(c_{Q}\right)\right|\left(\delta^{n+1} \lambda\right)^{\alpha}\left(\phi_{\varepsilon_{n} Q}\right) \leq C^{\prime} \eta^{\prime n}\left(\delta^{n+1} \lambda\right)^{\alpha} \sum_{Q \in G_{n+1}^{g}}\left(\phi_{\varepsilon_{n} Q}\right) .
$$

Now, we check the integrability of the majorant:

$$
\begin{aligned}
\int_{\mathbb{R}} \mid t & -\left.x\right|^{-\beta}\left|g_{n+1}(x)-g_{n}(x)\right| \\
& \leq C^{\prime} \eta^{\prime n}\left(\delta^{n+1} \lambda\right)^{\alpha} \sum_{Q \in G_{n}^{g}} \int_{\mathbb{R}}|t-x|^{-\beta} \phi_{\varepsilon_{n} Q}(x) d x \\
& \leq C^{\prime} \eta^{\prime n}\left(\delta^{n+1} \lambda\right)^{\alpha} \sum_{Q \in G_{n}^{g}}\left|t-x_{Q}^{*}\right|^{-\beta} \int_{\mathbb{R}} \phi_{\varepsilon_{n} Q}(x) d x \\
& \leq C^{\prime} \eta^{\prime n}\left(\delta^{n+1} \lambda\right)^{\beta} \sum_{Q \in G_{n}^{g}}\left|t-x_{Q}^{*}\right|^{-\beta} .
\end{aligned}
$$

Here $x_{Q}^{*}$ denotes a point of the support of $\phi_{\varepsilon_{n} Q}(x)$ closest to $t$. Using (37) and the fact that the distance between two different points $x_{Q}^{*}$ and $x_{Q^{\prime}}^{*}$ is at least $\delta^{n+1}$, we see that

$$
\begin{aligned}
\sum_{Q \in G_{n}^{g}}\left|t-x_{Q}^{*}\right|^{-\beta} & \leq 2 \sum_{k=0}^{\infty}\left(\varepsilon_{n} \delta^{n+1}+k \delta^{n+1}\right)^{-\beta} \\
& \leq 2 \delta^{(n+1)(-\beta)} \sum_{k=0}^{\infty}\left|\varepsilon_{n}+k\right|^{-\beta} \\
& \leq 2 \delta^{(n+1)(-\beta)}\left(\varepsilon_{n}^{-\beta}+\sum_{k=1}^{\infty} k^{-\beta}\right) \leq C \delta^{(n+1)(-\beta)} \varepsilon_{n}^{-\beta} .
\end{aligned}
$$


Substituting (39) in (38) and summing over all $n$, we get

$$
\int_{\mathbb{R}} \widetilde{g}(t) d t \leq C^{\prime \prime} \lambda^{-\beta} \sum_{n=1}^{\infty} \eta^{\prime n} \varepsilon_{n}^{-\beta}<+\infty .
$$

It remains to prove that $g$ satisfies Hölder's condition with the exponent $\alpha=\beta-1$. In fact this is a property of the potential $U_{\alpha}$ of any bounded function for which it is defined. To prove this, we take $t>0$ and write the following estimate:

$$
\left.\int_{\mathbb{R}}|| x\right|^{\alpha-1}-|x-t|^{\alpha-1} \mid d x=\int_{(-t ; 2 t)}+\int_{\mathbb{R} \backslash(-t ; 2 t)}=: J_{1}+J_{2} .
$$

We estimate each term:

$$
\begin{aligned}
& J_{1} \leq \int_{(-t ; 2 t)}|x|^{\alpha-1}+\int_{(-t ; 2 t)}|x-t|^{\alpha-1}=\frac{2}{\alpha}\left(1+2^{\alpha}\right) t^{\alpha} \\
& J_{2} \leq 2 \int_{(t ;+\infty)} x^{\alpha-1}-(x+t)^{\alpha-1} \leq 2(\alpha-1) \int_{(t ;+\infty)} t x^{\alpha-2} \leq 2 t^{\alpha} .
\end{aligned}
$$

When estimating $J_{2}$, we have used the inequality

$$
|h(x+t)-h(x)| \leq t \sup _{s \in(x, x+t)}\left|h^{\prime}(s)\right|
$$

for a smooth function $h$. The above estimates lead to the inequality

$$
\begin{aligned}
\left|\left(U_{\alpha} f\right)(t+\delta)-\left(U_{\alpha} f\right)(t)\right| & \leq \sup _{\mathbb{R}}|f| \int_{\mathbb{R}}\left(|t+\delta-x|^{\alpha-1}-|| t-\left.x\right|^{\alpha-1} \mid\right) d x \\
& \leq C(\alpha)\left(\sup _{\mathbb{R}}|f|\right) \delta^{\alpha} .
\end{aligned}
$$

The theorem is proved.

Remark 1. In the proof of the smoothness estimate for $g$ we have not used all the information we had about $f$. In fact, besides being bounded and belonging to the domain of $U_{\alpha}, f$ satisfies Hölder's condition with some exponent $r>0$. Using this fact and the well-known techniques of estimating operators similar to Rietz potentials (see, e.g., [8), we can check that $g$ satisfies Hölder's condition with the exponent $\beta-1+r$.

Remark 2. The theorem proved in [5] states that for the potentials $U_{\alpha}$ with $-1<\alpha<0$, uniqueness occurs if the density $g$ belongs to $C^{1+\varepsilon}$ with some $\varepsilon>0$. Theorem 2 shows that the latter condition cannot be replaced by Hölder's condition with the exponent $-\alpha$. So, there is a gap between the two results, which reduces as $\alpha \rightarrow-1$. If $\alpha \leq-1$, then no supplementary smoothness condition is needed: the mere existence of the potential suffices (see, e.g., [6]).

For the cases where $\alpha=0$ or $\alpha>1$ (except for the odd integers, for which uniqueness does not occur in any sense), the question as to whether the smoothness conditions imposed in [5] can be lifted remains open.

\section{§11. Extension of the Results to the multidimensional CASE}

The result of Theorem 1 can be extended to the case of Riesz potentials in spaces $\mathbb{R}^{d}$ with $d>1$. In this case, for $\alpha \in(0, d)$ we consider the set of all measurable functions $f$ satisfying the condition

$$
\int_{\mathbb{R}^{d}} \frac{|f(x)|}{1+|x|^{d-\alpha}} d x<+\infty
$$

(as above, we denote this set by $\operatorname{dom} U_{\alpha}$ ). We put

$$
U_{\alpha} f:=f *|x|^{d-\alpha}, \quad f \in \operatorname{dom} U_{\alpha},
$$


where $*$ denotes convolution in $\mathbb{R}^{d}$. Of major interest is the case where $d=2$ and $\alpha=1$ (Newton's potential of a charge concentrated in the plane). Note, however, that for $d>1$ there are no analogs of the uniqueness theorem mentioned in the Introduction.

The following generalization of Theorem 1 is true.

Theorem 3. For any $d \in \mathbb{N}$ and any $\alpha \in(0, d)$ there exists a nonzero function $f: \mathbb{R}^{d} \rightarrow$ $\mathbb{R}, f \in \operatorname{dom} U_{\alpha}$, and a set $E \subset \mathbb{R}^{n}$ of positive Lebesgue measure such that $\left.f\right|_{E}=0$, $\left.U_{\alpha} f\right|_{E}=0$, and $f$ satisfies Hölder's condition with some positive exponent.

The proof of this theorem is quite similar to that of Theorem 1 . We only comment on some details differing in the multidimensional case.

We need an operator $W_{\alpha}$, "the inverse operator" to $U_{\alpha}$. The precise expression for this operator (see, e.g., [2, page 241]) does not matter for us; the only thing we need is that if we now denote the number $d+\alpha$ by $\beta$, then statements 1)-3) and 5) of Lemma 1 will still be valid.

The role of $I$ will be played by the cube $I^{d}$, and we shall consecutively divide it into congruent cubes with side $\delta^{n}$. Instead of "the cap" $\phi(x)$ we use the function $\phi(|x|)$.

The computations made in [2, page 241] show that Lemma 2 remains valid in the multidimensional case. Lemmas 3 and 4 can be deduced from the latter as above.

Since now $\beta=d+\alpha$, most of the computations in the multidimensional case will repeat those in the one-dimensional case word for word if we also replace the derivative by the gradient everywhere. So, because of statement 3) of Lemma 1 we shall still have (3), (11), (12), (13), and (14) (now for the cube $Q$ with sides parallel to coordinate axes the symbol $Q[a]$ denotes $Q \backslash Q^{\prime}$, where $Q^{\prime}$ is the cube obtained from $Q$ by homothety with the center $c_{Q}$ and the dilation factor $a$ ).

The remaining part of the construction is the same. We note that estimates (25) and (26), which play a key role in the proof of Lemma 6 and seem to depend on the dimension, in fact remain true in the same form.

Remark. Theorem 2 also extends to the multidimensional case: for $d<\beta<2 d$, there exists a nonzero continuous function $g: \mathbb{R}^{d} \rightarrow \mathbb{C}$ and a set $E$ of positive measure such that if $t \in E$, then $g(t)=0, \int_{\mathbb{R}^{d}} g(x)|t-x|^{-\beta} d x=0$. The latter integral converges absolutely for all $t \in E$. The function $g$ satisfies Hölder's condition with the exponent $\min \{\beta-d ; 1\} 4$ The only difference in the proof is estimate (39), where sums become multiple (of order $d$ ). They will still converge because $\beta>d$.

\section{REFERENCES}

[1] A. B. Aleksandrov and P. P. Kargaev, Hardy classes of functions harmonic in the half-space, Algebra i Analiz 5 (1993), no. 2, 1-73; English transl., St. Petersburg Math. J. 5 (1994), no. 2, 229-286. MR:1223170 (94h:42034)

[2] D. B. Beliaev and V. P. Havin, On the uncertainty principle for M. Riesz potentials, Ark. Mat. 39 (2001), 229-243. MR 1861059 (2003a:31002)

[3] I. Binder, A theorem on correction up to gradients of harmonic functions, Algebra i Analiz 5 (1993), no. 2, 91-107; English transl., St. Petersburg Math. J. 5 (1994), no. 2, 301-315. MR.1223172 (94d:31006)

[4] J. Bourgain and T. Wolff, A remark on gradients of harmonic functions in dimension $\geq 3$, Colloq. Math. 60/61 (1990), no. 1, 253-260. MR1096375 (92c:31012)

[5] V. P. Havin, The uncertainty principle for one-dimensional Riesz potentials, Dokl. Akad. Nauk SSSR 264 (1982), no. 3, 559-563; English transl., Soviet Math. Dokl. 25 (1982), no. 3, 694-698. MR0659763 (84j:31002)

\footnotetext{
${ }^{4}$ This smoothness estimate can be obtained by a simple method similar to that used in the proof of Theorem 2. By the techniques mentioned in the remark after Theorem 2, it can by proved that $g \in C^{\beta-d+r}$ (in the case where $\beta-d+r$ is an integer, we mean the corresponding Zygmund class).
} 
[6] V. Havin and B. Jöricke, The uncertainty principle in harmonic analysis, Ergeb. Math. Grenzgeb. (3), Bd. 28, Springer-Verlag, Berlin, 1994. MR1303780 (96c:42001)

[7] T. Wolff, Counterexamples with harmonic gradients in $\mathbb{R}^{3}$, Essays on Fourier Analysis in Honor of Elias M. Stein (Princeton, NJ., 1991) (C. Fefferman, R. Fefferman, and S. Wainger, eds.), Princeton Math. Ser., vol. 42, Princeton Univ. Press, Princeton, NJ, 1995, pp. 321-384. MR.1315554 (95m:31010)

[8] A. Zygmund, Trigonometric series. Vols. I, II, 3rd ed., Cambridge Univ. Press, Cambridge, 2002. MR1963498 (2004h:01041)

Mathematics and Mechanics Department, St. Petersburg State University, Universitetskil Prospekt 28, Staryi Peterhof, St. Petersburg 198504, Russia

E-mail address: k.izyurov@gmail.com

Received 8/FEB/2007

Translated by THE AUTHOR 